\title{
Phenotype characterization of embryoid body structures generated by a crystal comet effect tail in an intercellular cancer collision scenario
}

This article was published in the following Dove Press journal:

Cancer Management and Research

20 January 2012

Number of times this article has been viewed

\author{
Jairo A Diaz \\ Mauricio F Murillo \\ Department of Pathology, \\ Hospital Departmental Villavicencio, \\ Hospital Departmental Granada, \\ Medicine School, University \\ Cooperative of Colombia, \\ Villavicencio, Meta, Colombia
}

\begin{abstract}
Cancer is, by definition, the uncontrolled growth of autonomous cells that eventually destroy adjacent tissues and generate architectural disorder. However, this concept cannot be totally true. In three well documented studies, we have demonstrated that cancer tissues produce order zones that evolve over time and generate embryoid body structures in a space-time interval. The authors decided to revise the macroscopic and microscopic material in well-developed malignant tumors in which embryoid bodies were identified to determine the phenotype characterization that serves as a guideline for easy recognition. The factors responsible for this morphogenesis are physical, bioelectric, and magnetic susceptibilities produced by crystals that act as molecular designers for the topographic gradients that guide the surrounding silhouette and establish tissue head-tail positional identities. The structures are located in amniotic-like cavities and show characteristic somite-like embryologic segmentation. Immunophenotypic study has demonstrated exclusion factor positional identity in relation to enolase-immunopositive expression of embryoid body and human chorionic gonadotropin immunopositivity exclusion factor expression in the surrounding tissues. The significance of these observations is that they can also be predicted by experimental image data collected by the Large Hadron Collider (LHC) accelerator at the European Organization for Nuclear Research, in which two-beam subatomic collision particles in the resulting debris show hyperorder domains similar to those identified by us in intercellular cancer collisions. Our findings suggest that we are dealing with true reverse biologic system information in an activated collective cancer stem cell memory, in which physics participates in the elaboration of geometric complexes and chiral biomolecules that serve to build bodies with embryoid print as it develops during gestation. Reversal mechanisms in biology are intimately linked with DNA repair. Further genotype studies must be carried out to determine whether the subproducts of these structures can be used in novel strategies to treat cancer.

Keywords: phenotype, embryoid bodies, cancer complexes, geometric triangular chiral crystal complexes, enolase, human chorionic gonadotropin
\end{abstract}

\section{Introduction}

Cancer is, by definition, the uncontrolled growth of autonomous cells that eventually destroys adjacent tissues and generates architectural disorder, and also shows how a cell population undergoing density-dependent growth can lead to a chaotic dynamic biological system. However, this traditional concept cannot be totally true. In three well documented studies, we have demonstrated that cancer tissues produce order zones in a space-time interval. Furthermore, we have documented self-assembled geometric triangular chiral crystal complexes and a framework of invariant vascular collagen geometric attractors in cancer tissue, and have demonstrated how this system evolves over time. ${ }^{1,2}$ Fractal geometry enables spatial correction errors to embrace the chaotic 
system in a way that permits new structures to emerge, and as a result, an ordered self-assembly of embryoid bodies with neural differentiation in the final stage of development of cancer can be predicted. ${ }^{3}$

The observation of molecules in cancer tissue that resemble embryoid body structures in which we recognize traits of the primordial organization of human positional identity probably represents the collective activation of cancer cells with a stem memory signature that prints copies of organizer proteins in a reverse information system. At this point in our investigations, it is necessary to establish the morphologic patterns of these structures, which serve as guidelines for easy recognition and could help other researchers extract them in the context of bulky tumor proliferation and a disordered volume overload structure of hemorrhage and necrosis.

Conceptualization of cancer using embryologic tissues is not new. In the 19th and 20th centuries, several investigators (including Rudolf Virchow, Julius Cohnheim, and John Beard) proposed the so-called "embryonic rest" hypothesis of cancer development. They suggested that adult tissues may contain embryonic remnants that normally lie dormant, but can be activated to become cancerous. Based on this theory, cancer may develop in populations of cells that are left in a dormant state in developing organs during embryogenesis. ${ }^{4}$ These were concepts that were too advanced for that time, generated controversy, and were rejected by scientists. ${ }^{5}$

Here the mandatory reflection is how is the process of cancer, which is responsible for so many deaths, linked to the process of birth? Both produce fetal oncoproteins and human chorionic gonadotropin, the hormone of pregnancy and development, and also have the chemical and physiologic properties of growth factors, and is a common phenotypic characteristic of cancer. According to the literature, a wide variety of malignant tumors express human chorionic activity. ${ }^{6-11}$ Under this premise and to decode the meaning of these structures in tumor biology, our goal was to determine whether we can identify the expression of human chorionic gonadotropin simultaneously with the presence of embryoid bodies in tumor tissues.

From the physics point of view, our documented images show that behind the genesis of these embryoid bodies there is a physical pregnancy platform space structured by geometric triangular chiral crystal complexes. Biology and physics are complementary subjects, and physicists have started to think about how physics governs cell growth and shape. ${ }^{12}$

Theoretical physicists share an intuition that, at the deepest level, all physical phenomena have a beautiful mathematical structure. Mathematically, physical theory describes these forces and particles as the dynamics of elegant geometry. Elementary particles are not a random mishmash, but have striking patterns and interrelationships that can be depicted in a diagram corresponding to one of the most intricate geometric objects known to mathematicians. Despite this formulation, until now, physicists have not been able to see these shapes directly, but they can see their effects. ${ }^{13}$ In this way, investigators have designed experiments using the Large Hadron Collider (LHC), a particle accelerator used by physicists to study the smallest known particles, ie, the fundamental building blocks of all matter. ${ }^{14-17}$ By studying what happens when these particles collide, physicists learn about the laws of nature. The LHC was designed to accelerate subatomic particles known as protons to energies of 7 trillion electron volts apiece and then smash them together to create tiny fireballs, recreating conditions that last prevailed when the universe was less than a trillionth of a second old, which these machines can pack into their little fireballs the farther back in time they can go, closer and closer to the "big bang", and the smaller and smaller are the things they can see. The LHC could be the first machine capable of causing matter to travel backwards in time and have the ability to jump backwards and reappear in the future or past, ie, particles that travel back in time to appear before the collisions that produced them. In biology, we have documented selfassembled geometric triangular chiral crystal complexes and a framework of invariant collagen vascular geometric attractors in cancer tissues. Using our electro-optic collision model, we were able to predict and replicate all the characteristics of this geometric complex that connects a physical phenomenon with the signal patterns that generate biologic chaos. Intrinsically fractal geometry makes spatial correction errors embrace the chaotic system in a way that permits new structures to emerge, and as a result, an ordered self-assembly of embryoid bodies with neural differentiation at the final stage of cancer development is a predictable process, in accordance with these findings in cancer chaos system collision eventgenerated geometry, and makes the system jump backwards in time with the assembly of embryoid bodies. The results have dual significance, in that they are not only unique in the medical literature but also confirm that theoretical physicists are right in their predictions of geometry and show the leap back in time to when the systems came into collision. Physics and biology are inextricably bound together, and probably have been since the beginning of time. It is now imperative to investigate whether a particle accelerator system, such as the LHC, produces geometric patterns similar to those we have identified in intercellular cancer. 
Interdisciplinary collaboration in research leads to new solutions to problems through the integration of knowledge. A particular finding in a field of research can complement solutions under new perspectives in another field of knowledge. The interdigitation of our findings in cancer intercellular collisions with elementary knowledge in physics could result in standard geometric patterns. The authors decided to undertake a rigorous review of macroscopic and microscopic material in well-developed malignant tumors, in which 90 embryoid bodies were visualized, and 67 cases were identified to have enolase-positive staining. The review followed the same parameters that were established in the initial phase of the investigation but with a new component, ie, visualization and documentation of the most representative hot spots of phenotypic embryoid body characterization in a wide range of epithelial and mesenchymal tumors.

Using selective immunopositivity of developing embryoid bodies to enolase, our obvious consideration was whether the surrounding tissues revealed immunoexpression of human chorionic gonadotropin in conjunction with these structures. Human chorionic gonadotropin is a glycoprotein consisting of two subunits, $\alpha$ and $\beta$, which are nonconvalently linked. This hormone is normally produced by syncytiotrophoblastic cells in the placenta and shows elevated levels in pregnancy. Its most important use is as a tumor marker in gestational trophoblastic disease and germ cell tumors. Human chorionic gonadotropin levels are occasionally elevated in other cancers.

\section{Materials and methods}

This study was approved by the ethics subcommittee of the Faculty of Medicine, University Cooperative of Colombia, and followed the guidelines of the Ministry of Health resolution 8430 of 1993, in accordance with the Declaration of Helsinki. All patients signed an informed consent form for use of their biological material for diagnostic and research purposes.

Samples for histopathology, cytopathology, and immunohistochemistry were taken from respective tissue samples and stained with hematoxylin and eosin, Papanicolaou, enolase, and human chorionic gonadotropin antibodies. To identify immunoexpression of human chorionic gonadotropin-related embryoid bodies, human chorionic gonadotropin immunostain labeling was carried out to compare the distribution, localization, and immunoreactivity of 67 formalin-fixed and paraffin-embedded tissue sections with the most representative hot spots of enolase-positive embryoid bodies from 90 previously analyzed samples.

\section{Collision model}

Although there is no accurate method for evaluation of collision processes in biological studies, it is possible to obtain indirect information via modeling other dynamic systems. Biology and physics can join forces to explore nature with microscopes, accelerators, and particle detectors. From the perspective of having methodologically and sequentially documented macroscopic and microscopic geometric patterns in malignant cancer tissues, we analyzed in close detail, step by step, the detector performance of the Compact Muon Solenoid (CMS), ie, compact because it is "small" for its enormous weight, muon for one of the particles it detects, and solenoid for the coil inside its huge superconducting magnet. CMS is designed to detect a wide range of particles and phenomena produced in high-energy collisions in the LHC. Resembling a cylindrical onion, different layers of the detector stop and measure different particles, and use these key data to build up a picture of events at the heart of the collision. Each particle that emerges is like a piece of a puzzle, with some pieces breaking up further as they travel away from the collision. Each particle leaves a trace in the detector and the task of the CMS is to gather up information about every particle, perhaps 20,100 , or even 1000 puzzle tracks, so that physicists can put the jigsaw together and see the full picture of what happened at the heart of the collision. Our goal setting records official real-time live images of European Organization for Nuclear Research experiment event 4285681 carried out using the LHC at $700 \mathrm{TeV}$ energy at 13:30 hours on March 30, 2010, in which two beams of subatomic particles called "hadrons" were collided headon at a very high recorded energy. Such animations based on real measures give physicists a good sense of which new particles can be detected and how long it will take to confirm them with sufficient statistical certainty.

\section{Statistical analysis}

We determined the embryoid body-selective distribution of neuron-specific enolase antibody immunopositivity, the relationship between HCG antibody immunostain positivity indexes and enolase-immunopositive embryoid transformation areas, and the relationship between an embryoid body pattern and a nonembryoid pattern with neuron-specific enolase antibody and human chorionic gonadotropin antibody immunostain. Chi-squares for the proportions were estimated using EPI-INFO software (version 6.04, Centers for Disease Control and Prevention, Atlanta, GA). 


\section{Results}

By detailed morphologic observation of these structures, we were able to find and generalize a series of constant morphologic features in different types of tumors. Embryoid bodies appear in all types of malignant lesions. These structures are intimately linked to displacement and migration of biological crystals in the extracellular matrix derived from intercellular collision of tumor cell membranes, and generate crystalejected comet tail effects with strange helicity states that arise in the form of spin domains (Figure 1A and B).

After a collision, high-density energy is released and a high temperature is created, with the acceleration process having a linear rise and decay time profile. In the late stages of the collision, energy is lost and the temperature decreases to an extreme low in relation to the stop particle friction interface with a high index of crystallization; at this point, there is a surge of a crystal-like hexagonal geometric pattern (Figure 1C-G). Our observations are correlated with recent physics collision findings. Researchers have demonstrated that weighted ping-pong balls colliding with a tube $600 \mathrm{~cm}$ (20 ft) long filled with polystyrene beads can reach a terminal velocity that allows the balls to continue sinking endlessly to an infinite depth. At this terminal velocity, structured hexagonal patterns have been observed ${ }^{18}$ (Figure $1 \mathrm{H}$ and I).

When the hexagon is assembled, the walls of the crystal structure trap molecules in their interior. In this closed environment, when the collision process stops, deceleration of the particles generates subcollisions and subejected crystal effect tails. It is at this particular moment that migration of crystals occurs in the interior of the hexagon embryoid bodies. It is conceivable from the biological point of view, that this process adds new amino acids to a chain reaction protein core with the creation of folding macromolecule adequate positional print identity (Figure $2 \mathrm{~A}-\mathrm{I}$ ). The point at which electromagnetism and biology converges is when the appearance of these structures is detected.

The morphogenesis of this embryoid body is attributable to scheduled short crystal migrations that act as molecular designers for the fine topographic map gradients that guide and sculpt the surrounding silhouette characteristics of the embryo body with surprising precision. These crystal migrations apparently generate the guidance ligand between cells that establishes
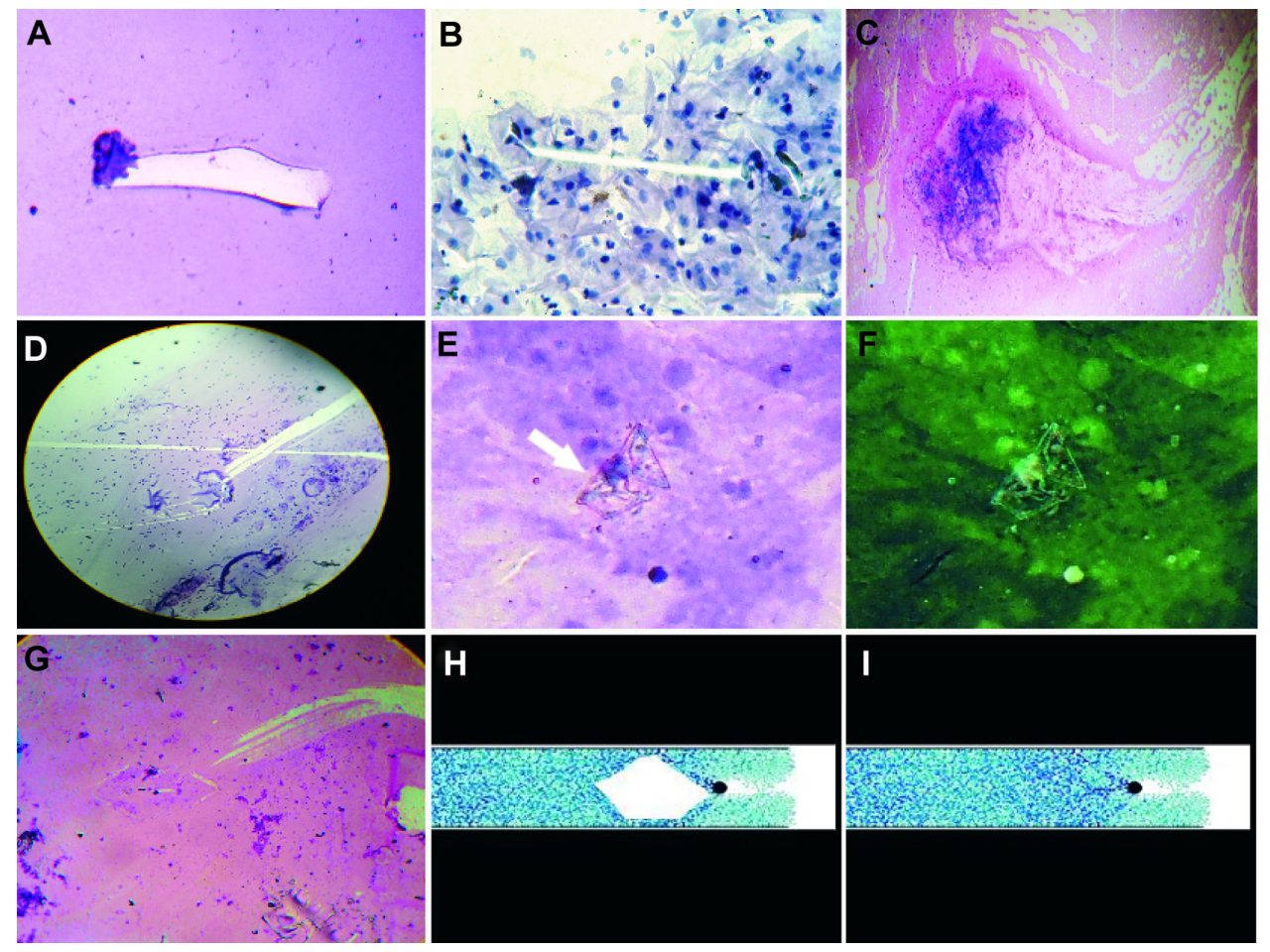

Figure I The genesis of embryoid bodies is intimately linked to displacement and migration of biological crystals derived from intercellular collision of tumor cell membranes. (A, B, C, and D) Migration of biological crystals in a case of lung adenocarcinoma, pleural fluid; case of cervical carcinoma in situ, vaginal smear; case of endometrial adenocarcinoma, endocervical smear and carcinoma of ovary and ascitic fluid, respectively. Papanicolaou staining (40x). (E and F) Embryoid bodies in a case of cervical carcinoma in situ, vaginal smear Papanicolaou staining (40x). (G) Crystal comet effect tail in a case of breast adenocarcinoma, fine needle aspiration, Papanicolaou staining (20x). (H) Detachment subimage of (I) image, which illustrates partial image taken from the projectile near the beginning of its trajectory as it falls through a tube filled with granular matter.

Note: Reprinted figure with permission from supplemental video of Pacheco-Vázquez et al. Phys Rev Lett. 106, 21800I (20II). Copyright 20II by the American Physical Society. Readers may view, browse, and/or download material for temporary copying purposes only, provided these uses are for noncommercial personal purposes. Except as provided by law, this material may not be further reproduced, distributed, transmitted, modified, adapted, performed, displayed, published, or sold in whole or part, without prior written permission from the American Physical Society. Available from: http://link.aps.org/doi/l0.1 103/PhysRevLett.106.21800I. 
A

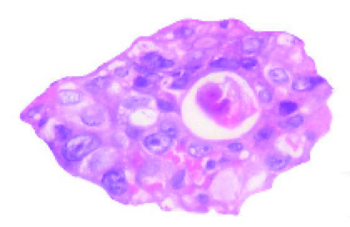

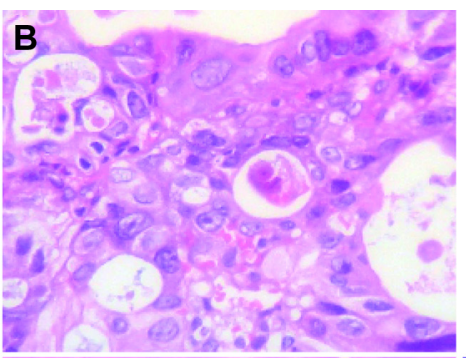

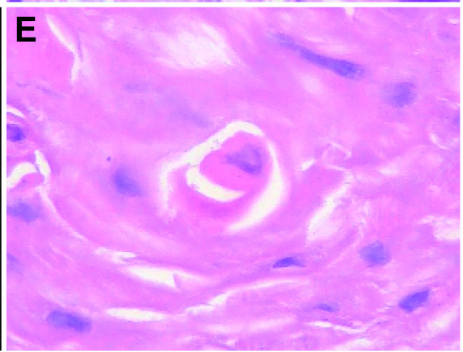

H

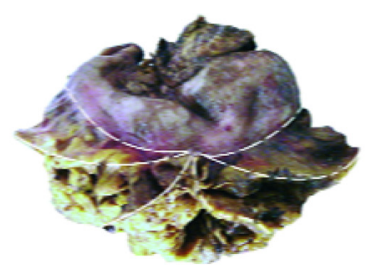

C
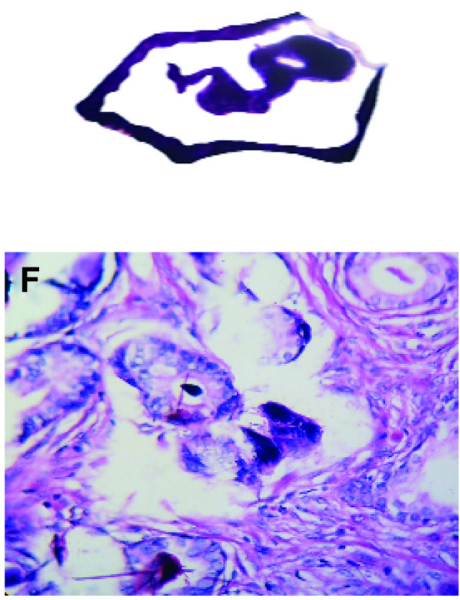

I

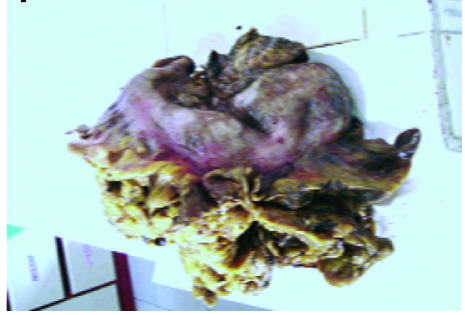

Figure 2 Crystals generate embryoid bodies in the interior of the hexagonal geometry. (A) Detachment subimage of (B) image of embryoid body self-assembly into a geometric triangular chiral hexagonal-like crystal complex in a case of colon adenocarcinoma with hematoxylin and eosin staining (20x). (C) Detachment subimage of (D) image of embryoid body into hexagonal-like crystal complex in a case of lung squamous cell carcinoma with hematoxylin and eosin staining (20x). (E,F) Embryoid body self-assembly in a case of prostate adenocarcinoma with hematoxylin and eosin staining (40x). (G) Well defined embryoid formation in a case of Grade I cervical intraepithelial neoplasia with hematoxylin and eosin staining (20x). ( $\mathbf{H}$ and I) Macroscopic representation of an embryoid body assembled into a geometric hexagonal template platform in a case of gastric adenocarcinoma.

head-tail positional identities (Figure 3A-I). The structures are located in a time-space interval within amniotic cavitylike domains, which are similar to a cordon (Figure 4A-I). The structures have the ability to generate mirror and fractal images (Figure 5A-I). Embryoid bodies show characteristic somite-like embryologic segmentation (Figure 6A-I). In our observations, the phenotype was more relevant, and a frequent characteristic was the positional identity within the crystal at the site of the optic placode assembled from the trajectory of the crystal that migrates from different positions and converges in the optic cavity, similar to somite-like structures.

\section{Inmmunophenotyping}

Statistical analysis determined that 90 embryoid patterns had high immunopositivity for neuron-specific enolase in 67 cases (75\%, $\left.\chi^{2}=17.16, P=0.000034\right)$, which was negative in 23 cases (25\%, Table 1). ${ }^{3}$ All 67 tissue-labeled sections were positive in relation to exclusion factor. Among the 67 tissuelabeled sections in which embryoid bodies showed immunopositivity exclusion factor in relation to enolase, 50 showed human chorionic gonadotropin immunopositivity and 17 were negative, indicating that, in labeled tissues, expression of enolase is limited to the structure and excludes the surrounding tissue. Similarly, human chorionic gonadotropin expression was negative with respect to the structure and positive for the surrounding tissue (Figure 7A-I). Localization of human chorionic gonadotropin immunopositivity is related to the tissues of the surrounding embryoid body and the walls of the triangular and hexagonal template platform where the structures are located (Figure 8A-F). According to the null hypothesis, the variables are independent. Based on the chi-square test which yielded a chi value $=38.61, \mathrm{df}=1, P<0.0001$, and an alpha of 0.05 , the null hypothesis was rejected (Table 2).

\section{Negative control}

To determine immunopositivity in the negative control, we took another 90 tissue-labeled examples without an embryoid body pattern. The enolase-immunopositivity relationship between 90 embryoid body pattern and 90 nonembryoid body pattern samples was analyzed in a total of 180 tissue slides. 

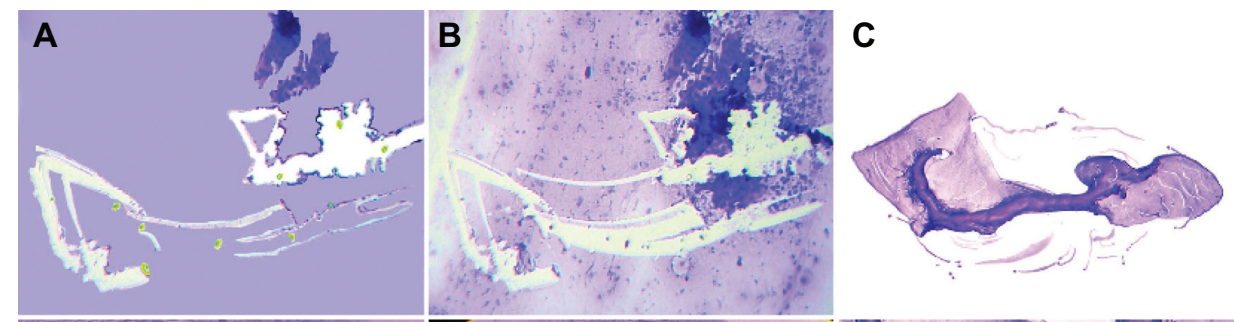
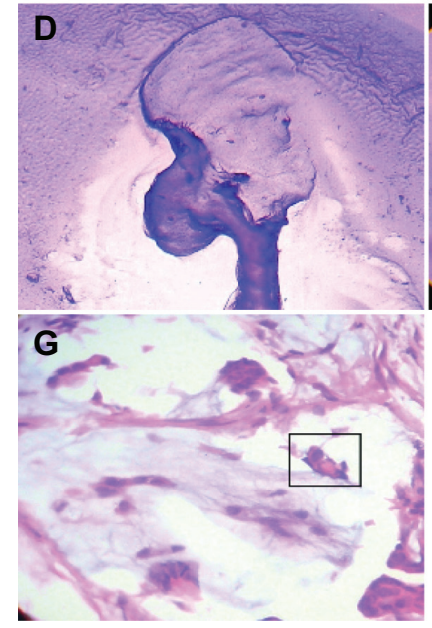

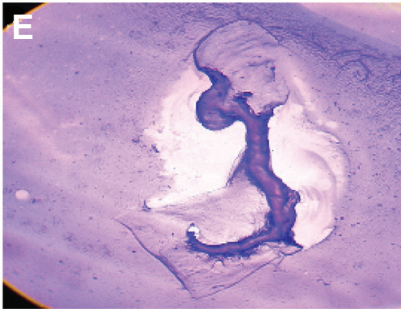

H

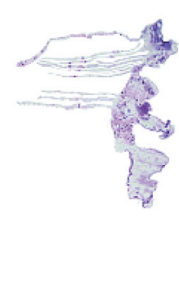

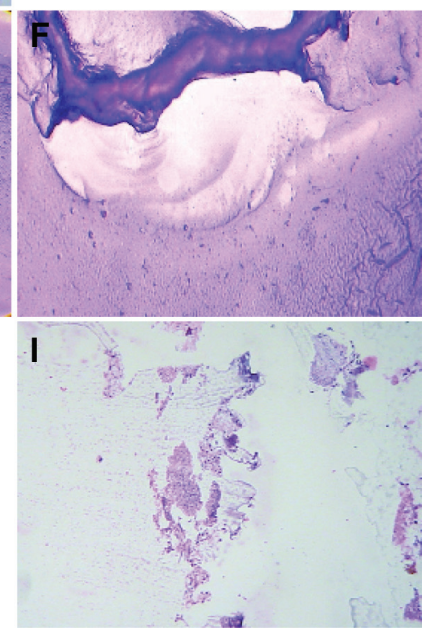

Figure 3 Close-up observation of scheduled short crystal migrations that guide and sculpt the surrounding silhouette characteristics of the embryo body with surprising precision. (A) Detachment subimage of (B) image, demonstrating geometric template platform guided by crystal displacement establishing head, tail positional identity in a case of endometrial adenocarcinoma, endocervical smear, Papanicolaou staining (40x). (C) Detachment panoramic subimage of (D, E, and F) which reveals in detail how short crystal migrations sculpting the surrounding embryoid body silhouette establish positional tissue ligands in a case of peritoneal carcinomatosis ascitic fluid. Papanicolaou staining (40 $\times$ ). (G) Well defined embryoid body in a case of breast adenocarcinoma with hematoxylin and eosin staining (20x). (H) Detachment subimage of (I) image which reveals embryoid body segmentation generated by massive crystal synchronic migrations in a case of undifferentiated necrotic tumor with hematoxylin and eosin staining (20x).
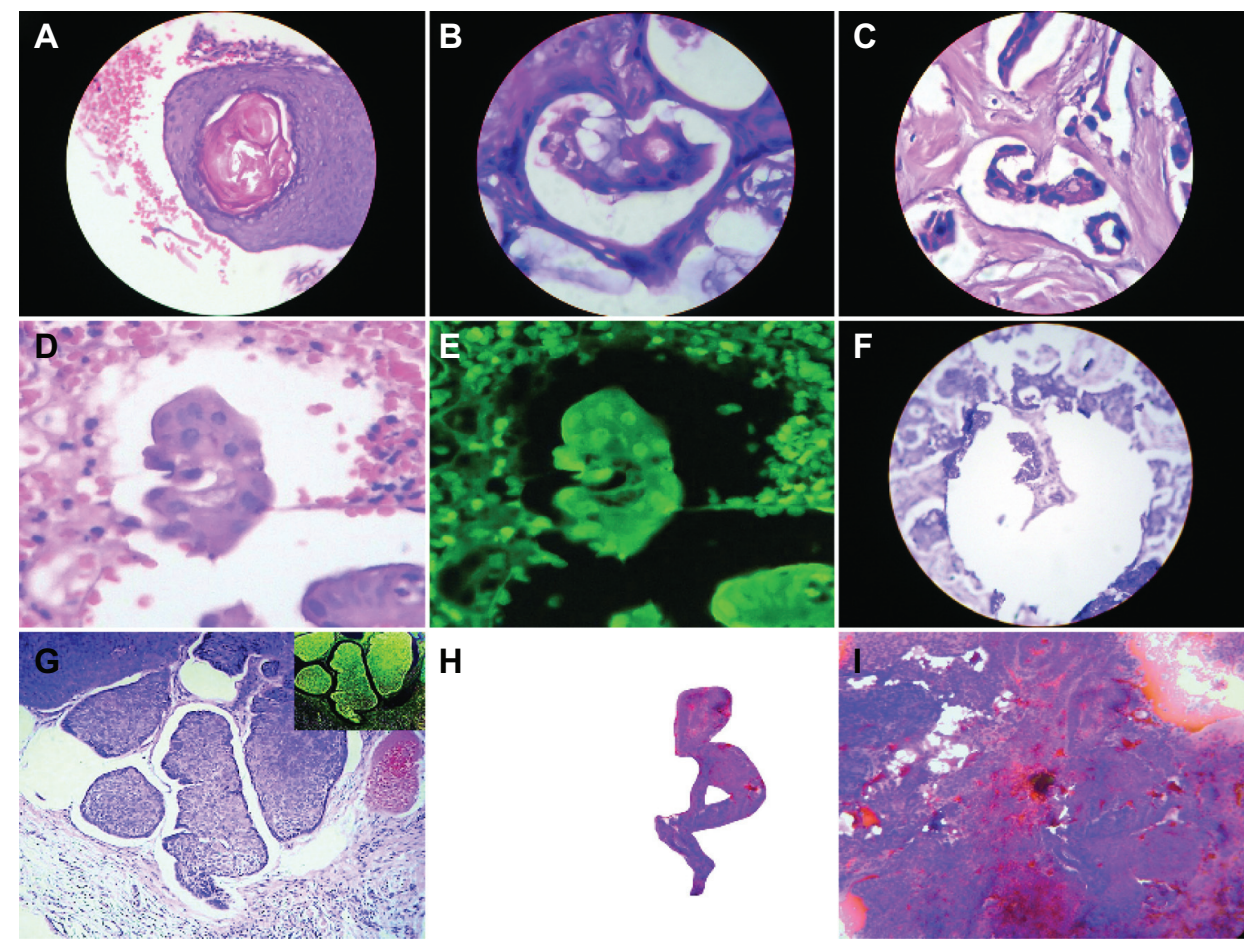

H
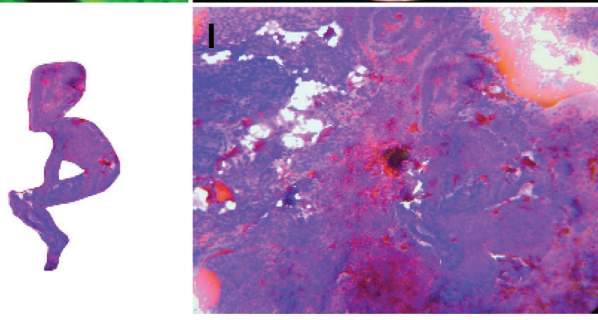

Figure 4 Embryoid structures are located in amniotic-cavity-like domains. (A) Skin squamous cell carcinoma, hematoxylin and eosin staining (20x). (B) Colon adenocarcinoma, hematoxylin and eosin staining (40x). (C) Breast adenocarcinoma, hematoxylin and eosin staining (40x). (D and E) Skin basal cell carcinoma, hematoxylin and eosin staining (40x). (F) Gastric adenocarcinoma hematoxylin and eosin staining (20x). (G) Skin basal cell carcinoma, hematoxylin and eosin staining (40x). (H) Detachment subimage of (I) Image of well-developed embryoid body in a case of endometrial adenocarcinoma, hematoxylin and eosin staining (20x). 
A

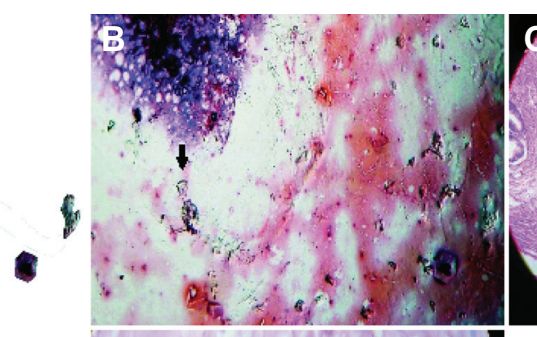

D

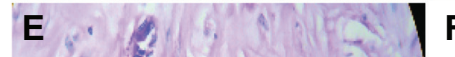

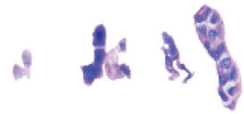

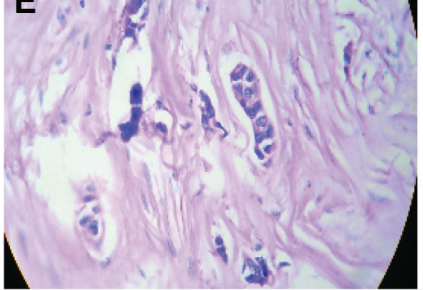

\section{$\mathbf{F}$}
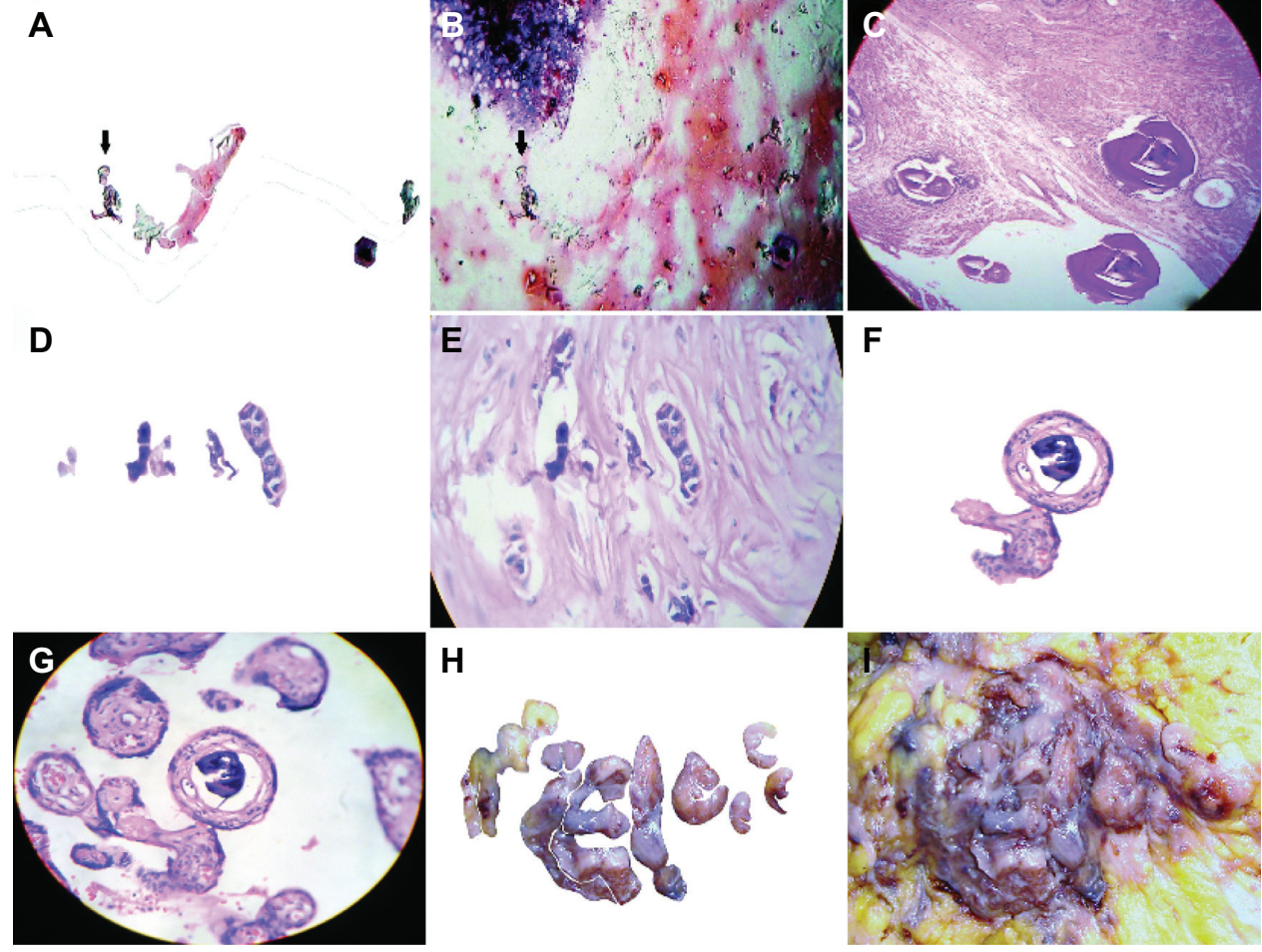

H
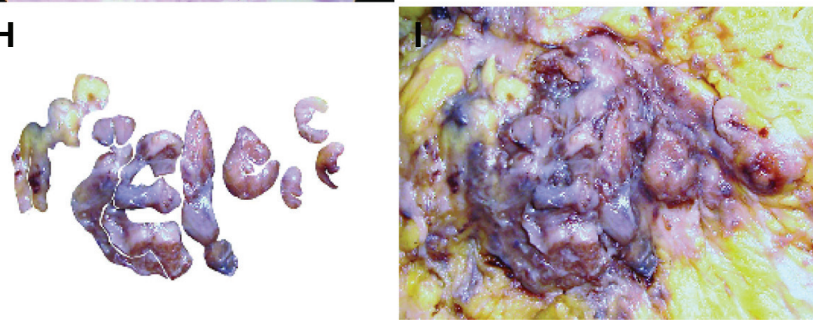

Figure 5 Embryoid bodies have the capability to generate fractal and mirror images. (A) Detachment subimage of (B) image revealing fractal components which converge in welldeveloped embryoid body in a case of peritoneal carcinomatosis ascitic fluid, Papanicolaou staining (20x). (C) Composite image that shows detachment mirror embryoid bodies taken from their original tissue position, in a case of prostatic intraepithelial neoplasia. (D) Detachment subimage of (E) showing alignment fractal copies of embryoid bodies in a case of breast adenocarcinoma, hematoxylin and eosin staining (20x). (F) Detachment subimage of (G) showing fractal embryoid within another one, in a case of gestational trophoblastic disease, hematoxylin and eosin staining (20X). (H) Detachment subimage of (I) showing macroscopic fractal components of embryoid bodies in a case of breast adenocarcinoma .
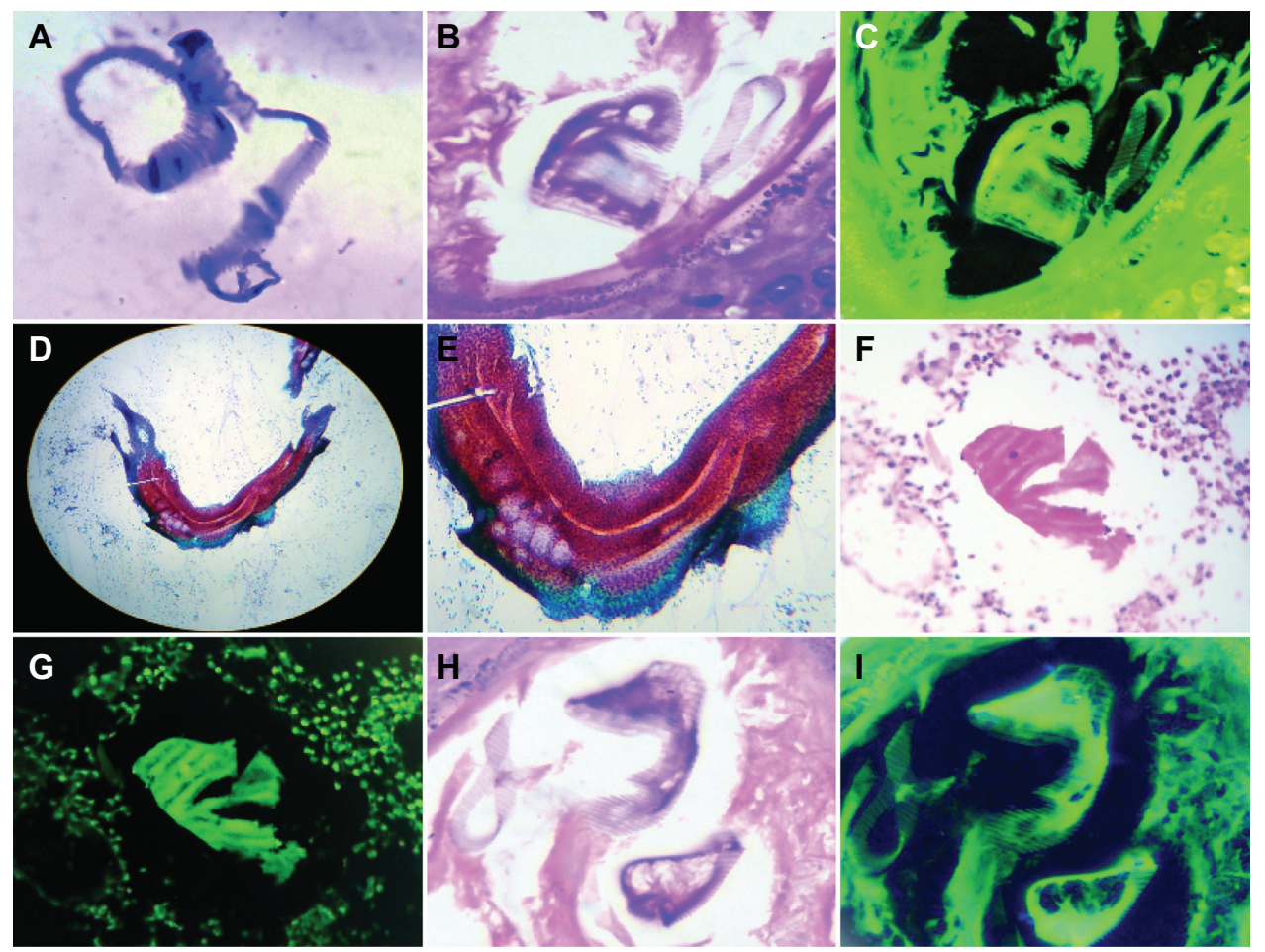

Figure 6 Embryoid bodies showing somites characteristic of embryologic segmentation, identified in (A), showing a case of peritoneal carcinomatosis ascitic fluid, Papanicolaou staining $(40 \times)$. (C) Negative image of (B) in a case of malignant eccrine poroma. (D and F) Somite-like segmentation in an embryoid body found in a case of papillary thyroid carcinoma, fine needle biopsy, Papanicolaou staining (40x). (G) Negative image of (F) in a case of gastric adenocarcinoma, hematoxylin and eosin staining (20x). (I) Negative image of $(\mathbf{H})$ in a case of colon adenocarcinoma, hematoxylin and eosin staining $(40 \times)$. 
Table I Embryoid body selective distribution of neuron-specific enolase antibody immunopositivity

\begin{tabular}{lll}
\hline Embryoid body & Enolase-positive & Enolase-negative \\
\hline 90 & 67 & 23 \\
& $75 \%$ & $25 \%$ \\
\hline
\end{tabular}

The variables were independent. The chi-square test yielded a chi value $=76.60$ and a $P<0.0001$, so the null hypothesis was rejected (Table 3 ). The negative human chorionic gonadotropin immunostain control was enolase-positive for both the embryoid body pattern and for the nonembryoid body pattern. The variables were independent. The chi-square test yielded a chi value $=0.04$ and $P<0.8376$, so the null hypothesis was not rejected (Table 4). In fully developed tumors free of medical manipulation, eg, chemotherapy or radiotherapy, it is possible to identify spatial temporal organization that clearly resembles fetus-placenta positional identities in the tissues (Figure 8G-I).

The collision figures are self-explanatory. We compared our images of intercellular cancer collisions with those of the European Organization for Nuclear Research experiment event 4285681 carried out with the LHC using $700 \mathrm{TeV}$ energy at 13:30 hours on March 30,2010, in which two beams of subatomic particles called "hadrons" were collided headon at very high energy. In the resulting debris, we observed patterns very similar to those we had described for intercellular collisions, ie, particles ejected from the comet core of the system. When the speed-decay hexagons and triangular mirror images were structured in the space-time interface, fractal bodies were discernible at the end of the collision process, at a very early stage (Figure 9A-I).

\section{Discussion}

All systems in which particles are in continuous dynamic movement comply with the laws of physics. This means that during the space-time interval of the life of any system, there exists the absolute potential to enter into a collision state at a given moment. In three previous studies, ${ }^{1-3}$ we have documented how intercellular cancer collisions generate geometric complexes, chirality, and fractals that serve as a pregnancy space-structural platform on which to build master embryoid bodies. The significance of these observations is

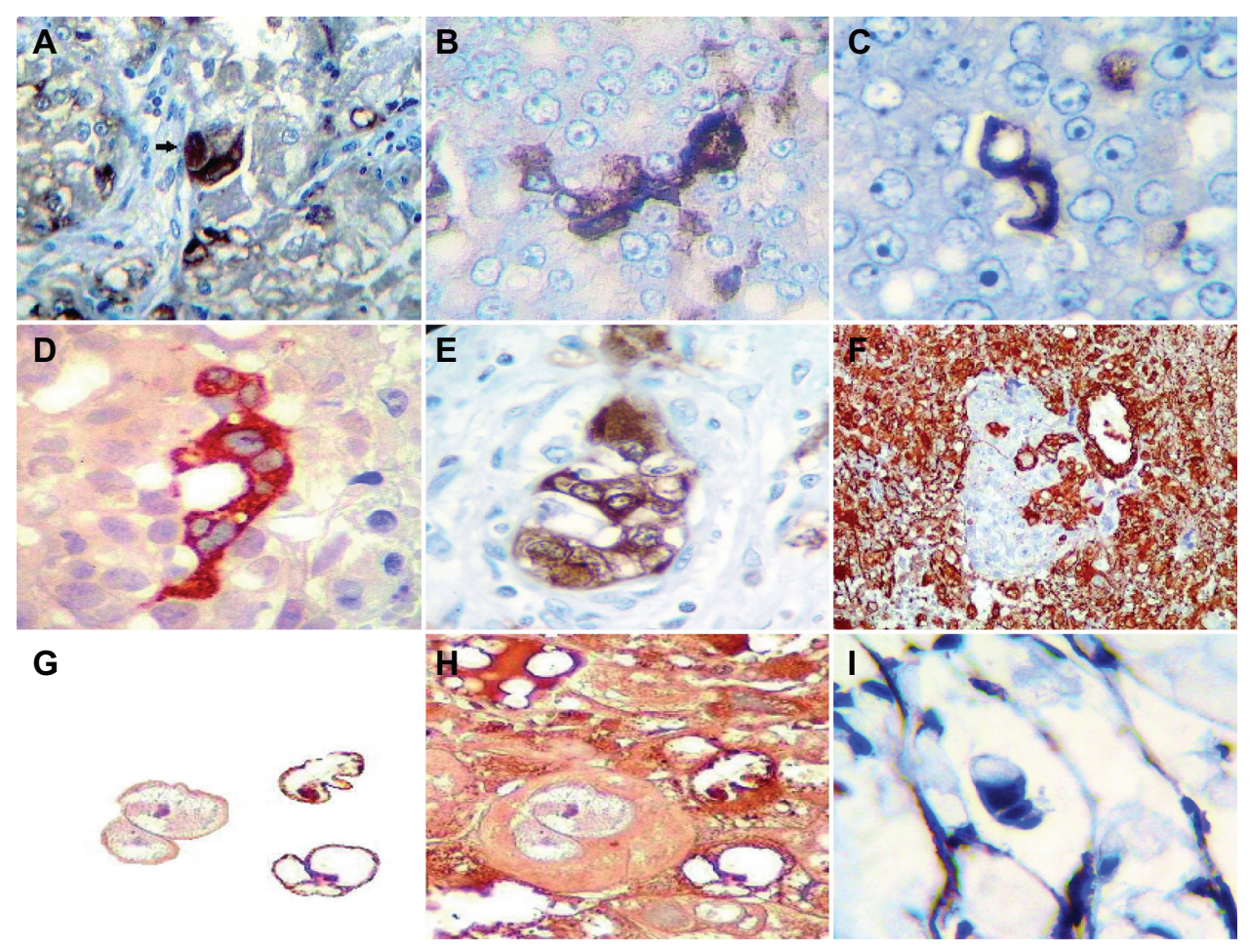

Figure 7 Immunostain demonstrated exclusion factor positional identity in relation to enolase immunopositivity expression of embryoid body and HCG immunopositivity exclusion factor identity expression in surrounding tissues; this means that in labeled tissues the expression of enolase is limited to the structure and excludes the surrounding tissue, just as the expression of HCG was negative to the structure and positive for the surrounding zone. Exclusion factor embryoid body enolase immunopositivity. (A) Case of lung carcinoma, (B and C) Case of liposarcoma, (D) Renal cell cancer, (E) Prostate adenocarcinoma, (F) Exclusion factor surrounding tissue HCG immunopositivity, case of lung carcinoma. Compare image (E) with (F). (G) Detachment subimage of $(\mathbf{H})$ Showing HCG immunopositivity of surrounding tissue and, embryoid bodies are negative in a case of trophoblastic testicular tumor. (I) HCG spot immunopositivity of surrounding tissue with immunonegativity of embryoid body in a case of liposarcoma. Abbreviation: HCG, human chorionic gonadotropin. 


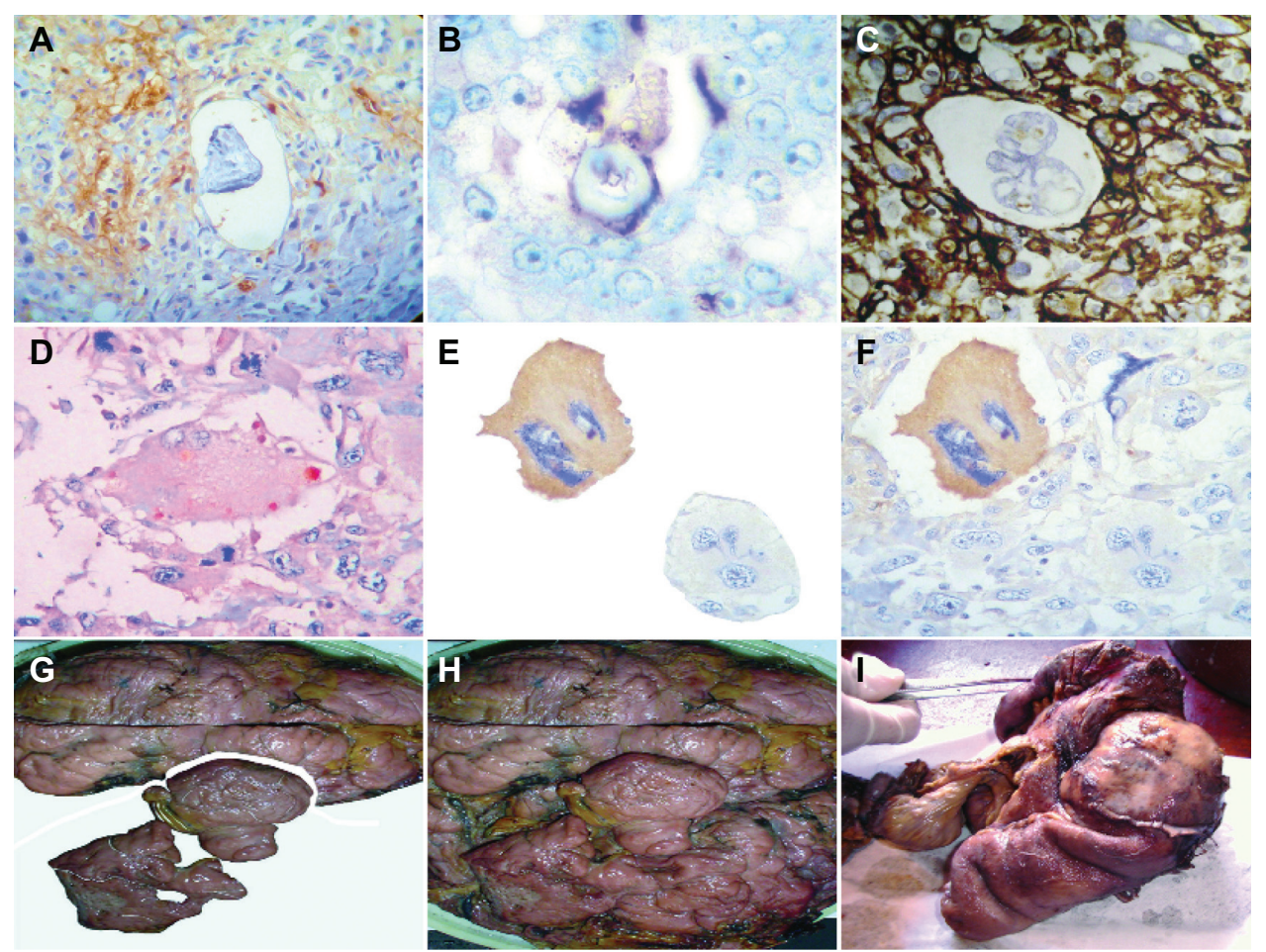

Figure 8 HCG immunopositivity localization related to embryoid body surrounding tissues and the walls of the triangular and hexagonal template platform where the structures are located. (A) HCG immunopositivity localization around inmmunonegativity embryoid body in a case of colon adenocarcinoma. (B) HCG-selective immunopositivity of the hexagonal template walls on which embryoid body is assembled in a case of undifferentiated sarcoma tumor. (C) HCG immunopositivity of surrounding tissue with inmmunonegativity of embryoid body structured inside hexagonal template platform in a case of renal cell carcinoma. (D) HCG selective spot walls immunopositivity of hexagonal geometric pattern in a case of papillary thyroid carcinoma. (E) Detachment subimage of (F) showing HCG selective immunopositivity of malignant giant cell in which two negative embryoid bodies are structured in their interior. Neighboring giant cell showing HCG immunonegativity, in a case of malignant fibrous histiocytoma. In Macroscopic fully developed malignant tumors is possible to identify spatial temporal organization that clearly reminds tissues fetus-placenta positional identities. (G) Detachment subimage of $(\mathbf{H})$ a case of leiomyosarcoma. (I) Case of colon adenocarcinoma.

Abbreviation: HCG, human chorionic gonadotropin.

that we were able to identify similar geometric patterns in the image data collected at the European Organization for Nuclear Research by the LHC accelerator. The LHC detector has a sophisticated electronic trigger system that precisely measures the time passage of a particle to accuracies in the region of a few billionths of a second. The trigger system also registers the location of the particles to millionths of a meter. This incredibly rapid and precise response is essential for ensuring that the particle is recorded in successive layers of the detector. However, we speculate whether these sophisticated detectors could identify geometric complexes and embryoid body print patterns.

Table 2 Relationship between HCG antibody immunostain positivity indexes in correlation with enolase immunopositivity embryoid transformation areas

\begin{tabular}{lll}
\hline $\begin{array}{l}\text { Embryoid body, } \\
\text { enolase-positive }\end{array}$ & HCG-positive & HCG-negative \\
\hline 67 & 50 & 17 \\
& $74.6 \%$ & $25.7 \%$ \\
\hline
\end{tabular}

Abbreviation: HCG, human chorionic gonadotropin.
The fact that both experiments demonstrated similar geometric patterns makes our findings even more intriguing. A geometric view of nature follows naturally from the way the world around us works. The simplest and most familiar examples are the forces of electricity and magnetism. In fact, theoretical physicists think that everything in the world, ie, all the forces of nature and even particles of matter, arise from different kinds of fields. The behavior of these fields hints at an underlying geometric structure in modern physics; the geometric objects associated with elementary particles are perfect and smooth-shaped, existing outside our space yet connected to it. Until now, physicists have not been able

Table 3 Negative control, relationship between positive embryoid body pattern and negative embryoid pattern

\begin{tabular}{lll}
\hline Tissue-labeled & $\begin{array}{l}\text { EB enolase- } \\
\text { positive }\end{array}$ & $\begin{array}{l}\text { Not EB enolase- } \\
\text { negative }\end{array}$ \\
\hline 180 & 67 & 9 \\
& $37.2 \%$ & $5 \%$ \\
\hline
\end{tabular}

Abbreviation: $\mathrm{EB}$, embryoid body. 
Table 4 Negative control, relationship between embryoid body pattern and nonembryoid body pattern with HCG distribution

\begin{tabular}{lll}
\hline $\begin{array}{l}\text { Positive enolase } \\
\text { tissue }\end{array}$ & $\begin{array}{l}\text { EB HCG } \\
\text { immune-positive }\end{array}$ & $\begin{array}{l}\text { Not EB HCG } \\
\text { immune-positive }\end{array}$ \\
\hline 76 & 50 & 7 \\
& $65.7 \%$ & $9.2 \%$ \\
\hline
\end{tabular}

Abbreviations: $\mathrm{EB}$, embryoid body; HCG, human chorionic gonadotropin.

to see these shapes directly, but have been able to see their effects.

The images we have been documenting in the course of our investigations are identical to those generated in the LHC experiments, and are not abstract curve graphics, mathematical formulas, or high-resolution computer simulations that only specialists understand. They are all real images that both specialists and nonspecialists can easily recognize, and are simple and straightforward.

The comparative image analysis of biology cancer collisions and the LHC collider experiment is self-explanatory, and the similarity observed is impressive. On the basis of this evidence, we can say, with absolute sense, that cancer intercellular collisions, a natural unique model, could reconcile until now elusive not observed exquisite geometric predicted by theoretical physicists, with the predictable replicable, visible order geometry documented by us in biological chaos system collisions. Theoretical physicists are correct in their predictions. If geometry represents the basic structural platform of the theory of everything, then crystals are the best interlocutors of this harmonic symphony. Interlocutors shared experience in their interaction history.

In physics, collision events should have the ability to jump backwards in time and reappear in the future, and past particles that travel back in time should appear before the collisions that produced them. Similar to a biology collision event, biomolecules can jump backwards like molecular time travel machines and generate primordial embryoid components normally produced in time before the collisions that produced them.

Furthermore, identification and documentation of similar patterns in dissimilar systems demonstrate that we
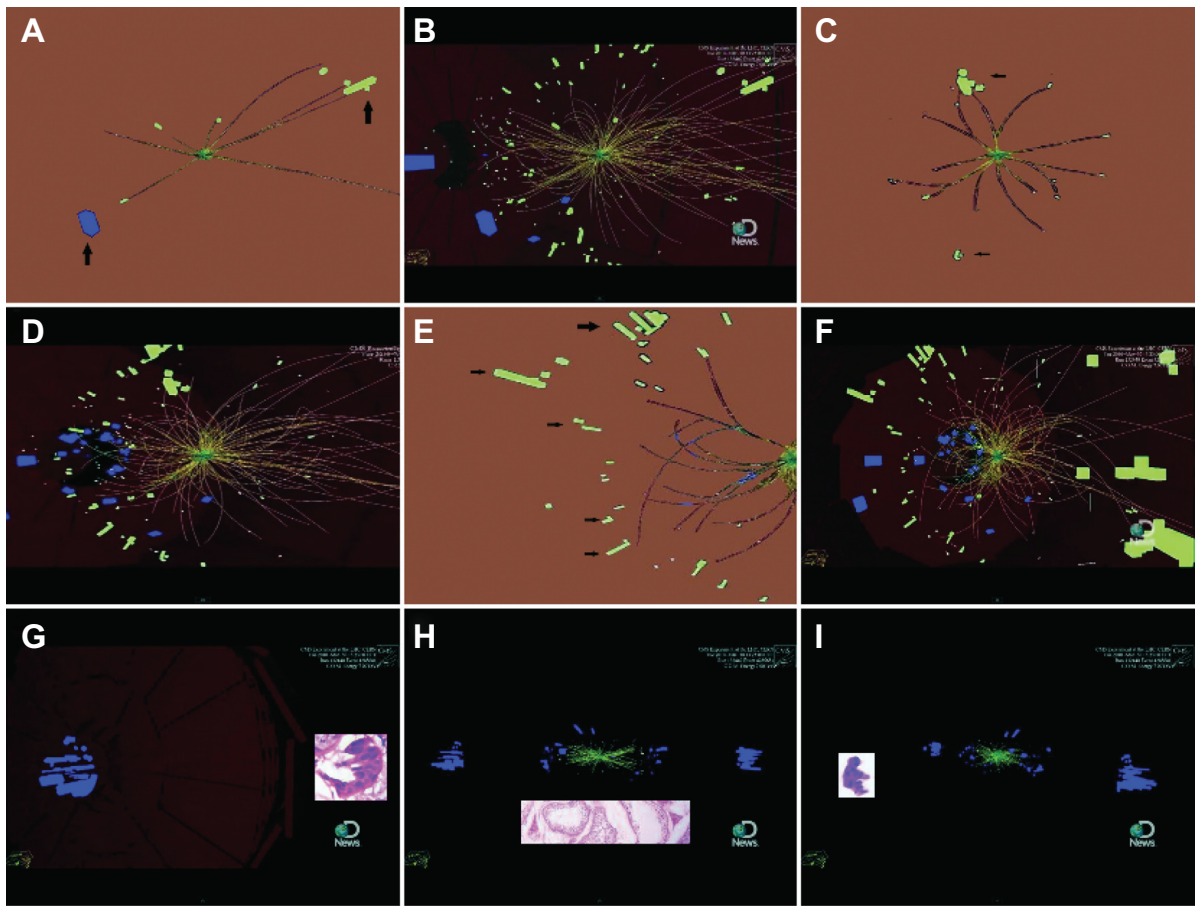

Figure 9 Images taken from video of a European Organization for Nuclear Research (CERN) experiment event 428568I carried out on March 30, 20I0 at I3:30 with 700 TeV energy at the Large Hadron Collider in which two beams of subatomic particles called "hadrons" are colliding head-on at very high energy. (A) Detachment subimage of (B) image which reveals in the resulting debris, self-assembled triangular chiral hexagonal geometric with ejected like comet tail pattern molecules. (C) Detachment subimage of (D) revealing polar chiral mirror images configuration linked by helicoidal pattern. (E) Detachment subimage of (F), showing fractal individual atoms and molecules converging in a configuration of positional head tail identities. (G) Close-up comparative image analysis between individual atoms and molecules integrated in positional head tail configuration in the end resulting debris of Large Hadron Collider with the microscopic self assembled embryoid body identified in intercellular cancer collisions. (H) Comparative image analysis between triangular chiral configuration of molecules in the resulting debris of large hadron collider and triangular mirror images self-assembly observed cancer intercellular collisions. (I) Comparative image analysis between embryoid bodies identified in cancer collisions and the final integration of individual molecules in head tail configuration positional identity. Note: Images reproduced from CERN movie 2010-076. Available from: http://cdsweb.cern.ch/record/I 256292.

Abbreviation: HCG, human chorionic gonadotropin. 
are confronted with standard laws of physics, and that the products of such events are noble structures, hyperorder domains, and unique indivisible molecules that remain unchanged at the atomic, molecular, and microscopic levels during transit. Thus, independent of the type of collision event, collisions generate geometry in a space-time interval. In the same way, in any given place, geometry has existed previously in the interaction area as a background of a collision event. This axiom is real and demonstrable, and is the transit in which a chaos system evolves to generate order, reaffirming that cancer tissues are an excellent natural model, with probably unique characteristics in which to analyze chaos states and molecular disorder. Collisions cause chaos, making invisible matter particles move, migrate, and become visible particles with a geometric, fractal, and chiral order signature imprinted on them at a very early stage. This is not a speculative issue, but a real fact that is possible to predict and replicate.

It is possible to identify this physical-biologic interface in intercellular cancer collisions. From a structural primordial one-dimensional platform, the crystal comet effect tails travel within the chaotic invisible matter and break the inertia of the matter, which generates movement and migration of molecules, causing them to become visible and generate fractal copies of crystal comet subunits that vibrate and grow to form a three-dimensional assembly. The fractal copies form a rain chain of crystals responsible for mapping and guiding moving molecules, and are expressed in physics as bioelectric and magnetic susceptibilities that sculpt the silhouette of the embryoid body by short scheduled crystal outflow migrations and probably govern the pathways of molecular communications that divide the embryoid body into segmental organizations or somite-like structures. This fact is in concordance with the notion that bioelectrical signals are necessary for normal head and facial formation in an organism. Recently, Tufts University biologists have reported for the first time that bioelectrical signals are necessary for normal head and facial formation in an organism, and have captured that process in a time-lapse video revealing never-before-seen patterns of visible bioelectrical signals outlining where the eyes, nose, mouth, and other features appear in an embryonic frog tadpole. ${ }^{19-21}$

A somite (or primitive segment) is a division of the body of an animal. In vertebrates, this is mainly discernible at the embryo stage. In the developing vertebrate embryo, somites are masses of mesoderm distributed along the two sides of the neural tube that will eventually become dermis (dermatome), skeletal muscle (myotome), or vertebrae (sclerotome). According to our observations, the phenotypic characteristic that is more common and relevant is the positional identity with crystal at the site of the optic placode, which is assembled from the trajectory of the crystal that migrates from different positions and converges in the optic cavity in direct relation to somite-like structures. Thus, identification of somite-like structures in these embryoid bodies is the fundamental observation that helps support our supposition that we are confronted with a completely self-assembled mesoderm neotissue.

Fractal geometry characterization makes spatial correction errors, embracing the chaotic system in a way that permits new structures to emerge, and chirality represents a certain type of protection mechanism. Here, it is necessary to mention the Meselson effect. There is evidence suggesting that asexual reproduction has allowed animals to evolve new proteins through the Meselson effect, which has helped them to survive better during periods of dehydration. The Meselson laboratory studies the evolution of asexuality in bdelloid rotifers. Meselson described the "Meselson effect," in which two alleles in an asexual organism evolve independently and divergently over time, producing essentially two genomes in one organism. However, what we observed was not two alleles but two well-developed structures in chiral position, signifying that at the molecular level, proteins in opposite positions might represent some type of protection of the system. ${ }^{22-24}$

From another point of view, immunophenotypic human chorionic gonadotropin characterization in malignant tissues is not new. The hormone of pregnancy and development, which also has chemical and physiologic properties of growth factors, is a common phenotypic characteristic of cancer. We believe that there exists interdependence between the positional immunopositivity of human chorionic gonadotropin and the development of the embryoid body in multiple ways, with the embryoid body having immunopositivity exclusion factor in relation to enolase and immunonegativity exclusion in relation to human chorionic gonadotropin. This is of hallmark significance, demonstrating that this structure plays an important role in the biology of the tumor and the regulatory effect that one tissue has over another, which is congruent with the literature indicating that extracted neural tissue incubated for 7-9 weeks with placental explants exhibits inhibitory effects upon $\beta$ human chorionic gonadotropin secretion. ${ }^{25}$ Human chorionic gonadotropin is defined as a negatively charged glycoprotein hormone produced by trophoblastic cells of the human placenta as well 
as by certain cancer cells. Human chorionic gonadotropin immunopositivity localization in surrounding tissues and the walls of the triangular and hexagonal template platform where the structures are located tells us that cancer cells around the body structure acquire trophoblastic differentiation. The early vertebrate germ embryo is surrounded by trophoblastic tissues, which are specialized devices developed by the germ to nourish the developing embryo. The analysis of these phenotype characteristics very clearly demonstrates that neotissue organization is not fortuitous or random, and is very complex. This is a noble and complete structural organization not previously documented in the cancer literature. At this point in time, the evidence suggests that, in tumoral biology systems, geometric attractor patterns constitute collider partners, emerging when a steady equilibrium state is disturbed. These attractor patterns probably govern how molecules self-assemble de novo, holding a vision of a new renaissance tissue.

Why is the tumor engaged in production and elaboration of these structures? The ability to understand such complex structures is important, and we welcome opinions from multidisciplinary fields of science. Duesberg et al have recently described their theory that cancer is just another form of speciation, the evolution of a new species, or xenogenesis. ${ }^{27}$ The idea that cancer represents evolution of a new species is not new, with various biologists hinting at it in the late 20th century. Julian S Huxley, the evolutionary biologist, in 1956 wrote "Once the neoplasy process has crossed the threshold of autonomy, the resultant tumor can be logically regarded as a new biologic species."

Last year, Dr Mark Vincent of the London Regional Cancer Program and University of Western Ontario argued that carcinogenesis and the clonal evolution of cancer cells are speciation events in the strict Darwinian sense.

Our hypothesis is that cancer arranges the molecules of geometry, chirality, and fractals to occupy the empty nest of the fractality lost by chronic inflammation, viral infection, and carcinogenic mutations. The objective is to return to the fractal order system. It is clear that fractal dynamics corrections can only be generated through millions of cell divisions, which represents the bulky mass of the process, but are absolutely necessary in the context of sustainability and nutrition support for these structures. Molecular microscopic diamonds, which make up the most natural diamonds, are formed under high-pressure and high-temperature conditions and existing at depths of 140-190 km (87-120 miles) in the Earth's mantle. Minerals provide the carbon source, and growth occurs over periods of 1.0-3.3 billion years.
These microscopic biological diamonds are the results of millions and millions of cellular divisions that are necessary to make the appropriate corrections. However, this process has an inherent tissue volume overload.

Our findings suggest that we are facing a true biological machinery of reversal system information. Recursive ontogeny recapitulates an earlier stage of development in collective cancer cells. Stem memory activation, which produces a replaced copy of "organizer proteins" generated by intercellular collisions in which physics participates in the elaboration of geometric fractal complexes hyperorder domains, chiral biomolecules, and ordered morphogenic differentiated proteins, acts as a template pregnancy space platform for building master bodies with embryoid print. Reversal mechanisms in biology are closely related to DNA repair. ${ }^{27}$ If cancer cells and stem cells share the same origin, ${ }^{28,29}$ then are we confronted with a repair system for the body to replenish damaged adult tissues? Further genotypic studies of these structures must be carried out to determine whether characterizing and isolating the exact cells or subproducts of these structures can be used in novel strategies to treat cancer.

\section{Acknowledgments}

The authors thank Dr Jesus Rosado and Dr Jorge Ovidio Cruz, executive directors of the Hospital Department of Villavicencio and Hospital Department of Granada, for their financial support of this research and help with logistics, and Miguel Novoa for his biostatistical analysis.

\section{Disclosure}

The authors report no conflicts of interest in this work.

\section{References}

1. Diaz JA, Jaramillo NA, Murillo MF. Geometric triangular chiral hexagon crystal-like complexes organization in pathological tissues biological collision order. PLoS One. 2007;2:e1282.

2. Diaz JA, Jaramillo NA, Murillo MF. Framework of collagen type I vasoactive vessels structuring invariant geometric attractor in cancer tissues: insight into biological magnetic fields. PLoS One. 2009;4:e4506.

3. Diaz JA, Murillo MF, Barrero A. Intercellular cancer collisions generate an ejected crystal comet tail effect with fractal interface embryoid body reassembly transformation. Cancer Manag Res. 2011;3:143-155.

4. Virchow R. Editorial Archive for pathologist. Anatomy and Physiology for Clinical Medicine. 1855;8:23-54. German.

5 Seaman W. The Cancer Problem. New York, NY: MacMillan; 1914.

6. Irles R. Beta-hCG expression by bladder cancers. Br J Cancer. 1989; 60:382-384.

7. Yamaguchi A, Ishida T, Nishimura G, et al. Human chorionic gonadotropin in colorectal cancer and its relationship to prognosis. $J$ Cancer. 1992;65:305.

8. Fukuyama M, Hayashi Y, Koike M. Human chorionic gonadotropin in gastric carcinoma. Virchows Arch A Pathol Anat Histopathol. 1987; 411:205-212. 
9. Yoshida J, Nagai K, Nishimura M, et al. Secretion of hCG/ $\beta$-hCG by squamous cell carcinoma of the lung in a 31-year-old female smoker. Jpn J Clin Oncol. 2000;30:163-166.

10. Nakanuma Y, Unoura M, Noto H, Ohta G. Human chorionic gonadotropin in primary liver carcinoma in adults. An immunohistochemical study. Virchows Arch A Pathol Anat Histopathol. 1986;409:365-373.

11. Syrigos KN, Fyssas I, Konstandoulakis MM, et al. Beta human chorionic gonadotropin concentrations in serum of patients with pancreatic adenocarcinoma. Gut. 1998;42:88-91.

12. Wolgemuth CW. Does cell biology need physicists? Physics. 2011;4(1):4.

13. Garret A, Owen JA. A Geometric theory of everything. Scientific American. 2010;303(6):54-61.

14. Khalil S. Search for supersymmetry at LHC. Contemporary Physics. 2003;44(3):193-201.

15. Belyaev A. Supersymmetry status and phenomenology at the Large Hadron Collider. Pramana. 2009;72(1):143-160.

16. Khachatryan AM, Sirunyan A, Tumasyan W, et al. Transverse momentum and pseudorapidity distributions of charged hadrons in pp collisions at $\sqrt{s}=0.9$ and $2.36 \mathrm{TeV}$. JHEP. 2010;2:41.

17. Brumfiel G. Beautiful theory collides with smashing particle data. Nature. 2011;471:13-14.

18. Pacheco-Vázquez F, Caballero-Robledo GA, Solano-Altamirano JM, Altshuler E, Batista-Leyva AJ, Ruiz-Suárez JC. Infinite penetration of a projectile into a granular medium. Phys Rev Lett. 2011;106:218001.

19. Vandenberg L, Morrie R, Spencer D. V-ATPase-dependent ectodermal voltage and ph regionalization are required for craniofacial morphogenesis. Dev Dyn. 2011;240:1889-1904.
20. Gomez C, Özbudak E, Wunderlich J, Baumann D, Lewis J, Pourquié O. Control of segment number in vertebrate embryos. Nature. 2008;454:335-339.

21. Pourquie O. Vertebrate somitogenesis. Annu Rev Cell Dev Biol. 2001;17:311-350.

22. Dequéant ML, Pourquie O. Segmental patterning of the vertebrate embryonic axis. Nature Reviews Genetic. 2008;9(5):370-382.

23. Arkhipova I, Meselson M. Transposable elements in sexual and ancient asexual taxa. Proc Natl Acad Sci U S A. 2000;97:14473-14477.

24. Welch M, Meselson M. Evidence for the evolution of bdelloid rotifers without sexual recombination or genetic exchange. Science. 2000;288:1211-1215.

25. Welch M, Meselson M. Karyotypes of bdelloid rotifers from three families. Hydrobiologia. 1998;387/388:403-407.

26. Shurtz-Swirski R, Simon RJ, Cohen Y, Barnea ER. Placenta human embryo modulates placental function in the first trimester; effects of neural tissues upon chorionic gonadotropin and progesterone secretion. Placenta. 1991;12:521-553.

27. Duesberg P, Mandrioli D, McCormack A, Nicholson JM. Is carcinogenesis a form of speciation? Cell Cycle. 2011;10:2100-2114.

28. Lindahl T, Barnes DE. Repair of endogenous DNA damage. Cold Spring Harb Symp Quant Biol. 2000;65:127-134.

29. Calvin S, Jin Y, Loudon WG, et al. Increase developmental plasticity of human keratinocytes with gene suppression. Proc Natl Acad Sci US A. 2011;108:12793-12798.
Cancer Management and Research

\section{Publish your work in this journal}

Cancer Management and Research is an international, peer-reviewed open access journal focusing on cancer research and the optimal use of preventative and integrated treatment interventions to achieve improved outcomes, enhanced survival and quality of life for the cancer patient The journal welcomes original research, clinical \& epidemiological

\section{Dovepress}

studies, reviews \& evaluations, guidelines, expert opinion \& commentary, case reports \& extended reports. The manuscript management system is completely online and includes a very quick and fair peerreview system, which is all easy to use. Visit http://www.dovepress.com/ testimonials.php to read real quotes from published authors. 